\section{La matrice extracellulaire}

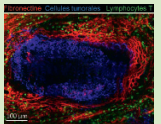

Un obstacle au contact entre lymphocytes $\mathrm{T}$ et cellules tumorales

Hélène Salmon, Emmanuel Donnadieu
Institut Cochin, Université Paris-Descartes, CNRS UMR 8104, Inserm U1016, département d'immunologie et d'hématologie, 22, rue Méchain, 75014 Paris, France. emmanuel.donnadieu@inserm.fr

\section{Les lymphocytes T sont localisés} préférentiellement dans le stroma des tumeurs

Les cellules tumorales expriment des antigènes reconnus par les lymphocytes T (LT) et peuvent, par conséquent, déclencher une réponse immunitaire antitumorale spécifique. Les LT doivent franchir différentes étapes pour mettre en place une réponse efficace: (1) être activés dans les ganglions drainant la tumeur par des cellules spécialisées leur présentant les antigènes tumoraux ; (2) entrer dans la tumeur à partir du sang; et (3) exercer leurs fonctions antitumorales, en particulier la lyse des cellules malignes. Toutefois, la tumeur

Vignette de l'article : fibronectine, cellules tumorales, lymphocytes T (๔ Emmamuel Donnadieu). peut échapper au contrôle immunitaire, et de nombreux mécanismes limitant les effets des LT ont été décrits, en particulier l'environnement immunosuppresseur que constitue la tumeur. Un autre obstacle, rarement pris en compte par les immunologistes, concerne la localisation des LT au sein même de la tumeur. Une tumeur solide ne se réduit pas à une masse de cellules malignes en division. En effet, les cellules malignes sont nichées dans le stroma tumoral, un environnement tissulaire associé à la tumeur. Ce stroma, qui entoure les îlots de cellules tumorales, est composé de fibroblastes, de cellules du système vasculaire et de cellules du système immunitaire, en association avec une matrice extracellulaire formée en particulier de fibres de fibronec-

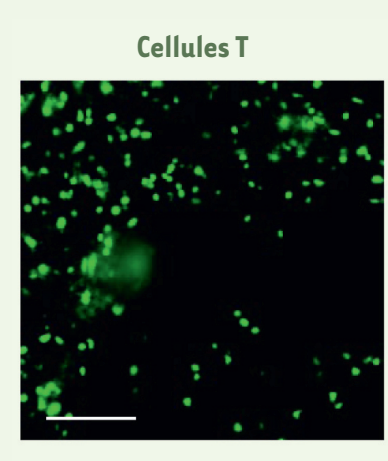

Cellules tumorales

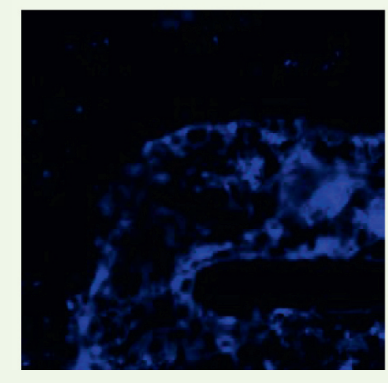

Fibronectine

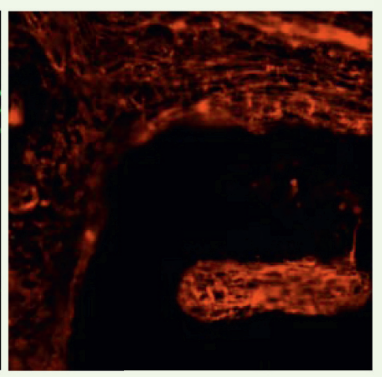

Superposition

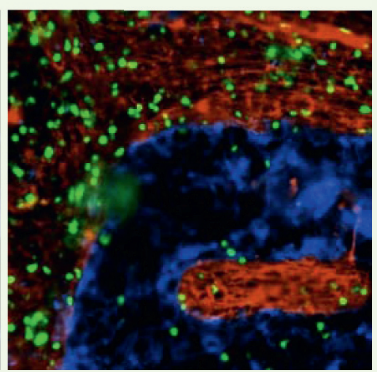
tine et de collagène. Or, dans les tumeurs en progression, les LT se trouvent, le plus souvent, non pas en contact direct avec les cellules tumorales, mais majoritairement dans le stroma [1]. Les cellules malignes étant

considérées comme les principales cibles des LT cytolytiques, la distribution inégale de ces derniers dans les tumeurs pourrait fortement limiter leurs fonctions antitumorales, et représenter un obstacle majeur à l'efficacité des immunothérapies utilisant les LT.

Dans le but d'identifier les mécanismes contrôlant la distribution intratumorale des LT, nous avons utilisé des techniques d'imagerie de fluorescence couplées à une préparation ex vivo de tranches de tumeurs pulmonaires humaines maintenues en survie. Dans un article publié récemment dans le Journal of Clinical Investigation [2], nous montrons que les LT déposés sur les tranches se comportent comme les LT déjà présents dans la tumeur: ils s'accumulent majoritairement dans le stroma et sont plus mobiles dans ce compartiment que dans les îlots tumoraux (Figure 1).

\section{Quels sont les facteurs limitant} I'infiltration des lymphocytes T dans les régions tumorales?

Différents mécanismes peuvent être à l'origine de l'incapacité des LT à infiltrer l'épithélium tumoral. Citons la contrainte physique que crée l'organisation dense et compacte des régions

Figure 1. Les lymphocytes T déposés sur des tranches de tumeurs pulmonaires humaines s'accumulent préférentiellement dans le stroma. Des LT purifiés du sang périphérique, activés in vitro, puis chargés avec un marqueur fluorescent (Hoechst, vert) ont été déposés sur une tranche de tumeur pulmonaire humaine marquée ensuite par des anticorps anti-fibronectine (protéine de la matrice extracellulaire présente dans le stroma, rouge), et anti-EpCAM (epithelial cell adhesion molecule) (marquant les cellules tumorales en bleu). La superposition des trois images apparaît dans la photographie en bas à droite. Barre d'échelle : $100 \mu \mathrm{m}$. 


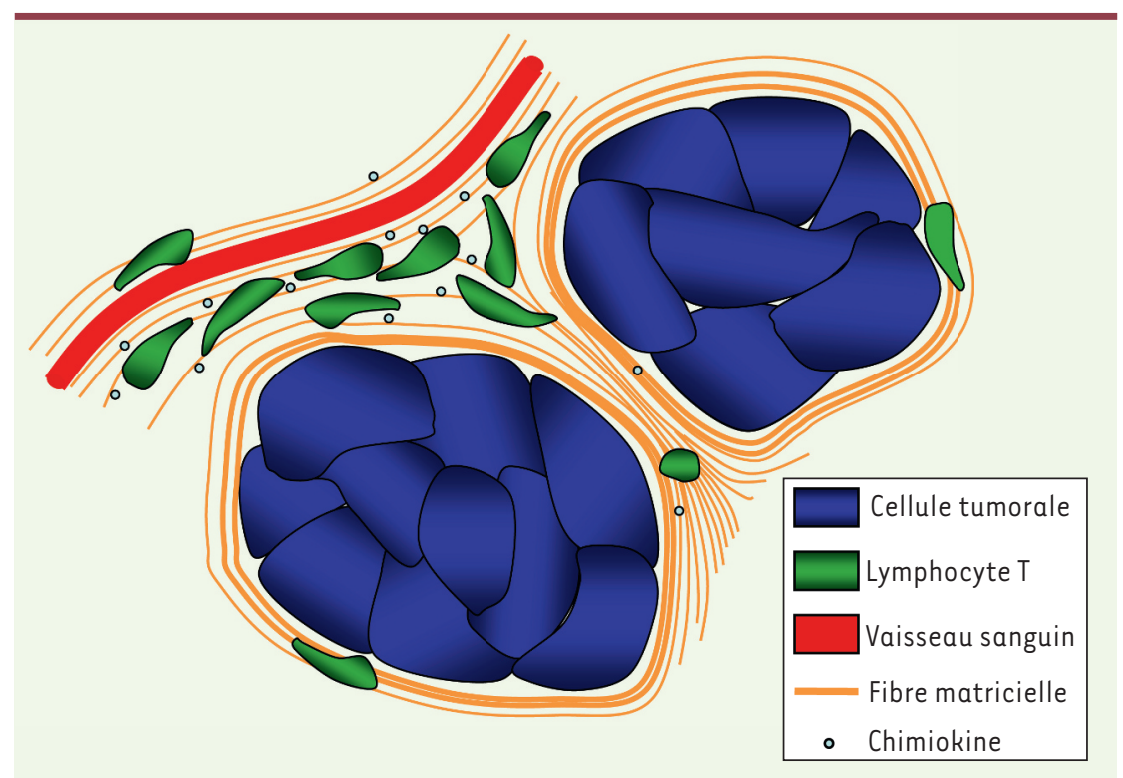

Figure 2. Dans les tumeurs pulmonaires humaines, les lymphocytes $T$ ont un accès limité aux régions tumorales. Représentation schématique de la distribution des LT dans une tumeur solide. Les LT s'accumulent préférentiellement dans des zones stromales caractérisées par la présence de chimiokines et une structure fibrillaire lâche, dans lesquelles ils sont mobiles. À l'inverse, la présence de fibres matricielles denses et linéaires autour des îlots tumoraux limite le contact des LT avec les cellules tumorales.

tumorales ainsi qu'un défaut dans l'expression de molécules chimio-attractantes [3]. Afin de déterminer si la densité des îlots tumoraux constitue une barrière physique à l'entrée des LT, nous avons utilisé un système de xénogreffe dans lequel les cellules tumorales ont été génétiquement modifiées pour exprimer la chimiokine CCL5 (CC chemokine ligand 5) avant d'être greffées chez des souris immunodéficientes. Dans ces conditions et à la différence de la situation contrôle, dans le système de tranches d'explants de tumeurs, les LT parviennent à infiltrer les îlots de cellules tumorales exprimant CCL5. Ces lymphocytes peuvent adapter leur morphologie à cette contrainte structurale, qui n'est donc pas une barrière absolue à l'infiltration lymphocytaire. Par ailleurs, ces expériences sont en accord avec différents travaux suggérant qu'une expression réduite de chimiokines par les cellules tumorales [3], voire une inactivation de ces chimiokines [4], pourrait également limiter la rétention des LT dans les îlots tumoraux.

\section{Pourquoi les lymphocytes T}

s'accumulent-ils préférentiellement dans le stroma des tumeurs en progression?

Dans les explants de tumeurs pulmonaires humaine, la distribution des LT ne se fait pas de façon homogène dans le stroma. Celui-ci est composé de différentes régions dans lesquelles la mobilité lymphocytaire est favorisée ou au contraire limitée. En visualisant les interactions entre les $L T$ et les autres éléments - cellulaires et structuraux - du tissu, nous avons pu corréler ces différents modes de migration à l'organisation particulière des fibres de la matrice extracellulaire dans le stroma. Une mobilité active, dépendante des chimiokines, est observée dans les zones pauvres en fibres de collagène et de fibronectine; au contraire les LT migrent faiblement dans les régions fibrillaires denses et s'y accumulent peu. L'observation de zones stromales promigratoires pour les LT confirme la présence, dans les tumeurs humaines pulmonaires, de structures lymphoïdes associées à un bon pronostic [5].
Outre leur espacement, la disposition des fibres matricielles est primordiale dans le contrôle de la mobilité des LT. Les régions périvasculaires, dont la structure fibrillaire est lâche et organisée, sont ainsi favorables aux mouvements des LT le long des vaisseaux. Par ailleurs, et de façon remarquable, des fibres denses et linéaires sont observées dans les régions stromales adjacentes aux îlots tumoraux. À cette interface stroma-tumeur, la majorité des LT présentent des trajectoires parallèles aux fibres, qui limitent ainsi leur accès aux régions tumorales. En résumé, l'architecture du stroma, en contrôlant le chemin migratoire des cellules T à l'intérieur des tumeurs et leur localisation, est susceptible d'influencer la réponse immunitaire antitumorale (Figure 2).

\section{Des molécules thérapeutiques} pourraient-elles favoriser l'infiltration des LT dans la masse tumorale?

La dérégulation de l'organisation de la matrice extracellulaire est caractéristique de nombreux cancers. La rigidification et le développement fibrotique qui en résultent et qui influencent la croissance et la prolifération des cellules tumorales sont corrélés à un mauvais pronostic pour le patient [6]. Notre étude souligne l'impact supplémentaire que représente l'organisation des fibres stromales qui influencent le comportement migratoire des LT et, en conséquence, leur distribution au sein de la tumeur. Étant donné le rôle négatif joué par une matrice extracellulaire dense, une question importante est maintenant de déterminer si la barrière fibrillaire peut être détruite pour améliorer l'accès des LT aux cellules tumorales. Nous avons montré qu'un traitement des tranches de tumeur avec la collagénase - une enzyme bactérienne altérant les fibres de collagène - réduit la densité de collagène et augmente le nombre de LT au contact des cellules malignes. Bien que cette enzyme ne puisse être utilisée en clinique en raison des 
effets secondaires qu'elle entraîne, nos résultats suggèrent que des traitements antifibrotiques seraient susceptibles de faciliter l'infiltration des LT dans les régions tumorales. Des études réalisées dans des modèles de tumeurs murines ont permis d'identifier plusieurs protéines responsables de la formation des fibres matricielles. C'est notamment le cas de la lysyl oxydase (LOX), une enzyme qui assemble les molécules de collagène en fibres linéaires et rigides, telles qu'on en observe dans le stroma réactif de tumeurs en progression. II est intéressant de noter que l'inhibition de l'activité enzymatique de LOX par des drogues pharmacologiques diminue à la fois l'apparition des fibres matri- cielles autour de la masse tumorale, mais aussi la croissance tumorale [7]. Ces dernières années, plusieurs composants du stroma tumoral ont été ciblés en thérapeutique : citons la vascularisation tumorale avec des anticorps dirigés contre le VEGF (vascular endothelial growth factor). La matrice extracellulaire, en raison des effets néfastes sur la capacité des LT à atteindre leurs cibles tumorales, représente, de ce fait, une nouvelle cible d'intérêt dans la lutte contre les cancers solides. $\diamond$

The extracellular matrix: an obstacle to $\mathrm{T}$ cell-tumor cell interaction

\section{LIENS D'INTÉRÊT}

Les auteurs déclarent n'avoir aucun lien d'intérêt concernant les données publiées dans cet article.

\section{RÉFÉRENCES}

1. Ohtani H. Focus on TILs: prognostic significance of tumor infiltrating lymphocytes in human colorectal cancer. Cancer Immun $2007 ; 7: 4$.

2. Salmon H, Franciszkiewicz K, Damotte D, et al. Matrix architecture defines the preferential localization and migration of T cells into the stroma of human lung tumors.J Clin Invest 2012 ; 122 : 899-910.

3. Pivarcsi A, Muller A, Hippe A, et al. Tumor immune escape by the loss of homeostatic chemokine expression. Proc Natl Acad Sci USA 2007 ; 104 : 19055-60.

4. Molon B, Ugel S, Del Pozzo F, et al. Chemokine nitration prevents intratumoral infiltration of antigen-specific T cells. J Exp Med $2011 ; 208$ : 1949-62.

5. Dieu-Nosjean MC, Antoine M, Danel C, et al. Longterm survival for patients with non-small-cell lung cancer with intratumoral lymphoid structures. J Clin Oncol $2008 ; 26: 4410-7$.

6. Lu P, Weaver VM, Werb Z. The extracellular matrix : a dynamic niche in cancer progression. J Cell Biol 2012 ; $196: 395-406$.

7. Levental KR, Yu H, Kass L, et al. Matrix crosslinking forces tumor progression by enhancing integrin signaling. Cell $2009 ; 139: 891-906$.

\section{NOUVELLE}

\section{Tolérance maternelle du fœtus}

\section{Le rôle d'un enhancer de Foxp3 lors de l'émergence des mammifères euthériens}

Laure Coulombel médecine/sciences, 2 rue d'Alésia, 75014 Paris, France.

laure.coulombel@inserm.fr
> Chez les mammifères, l'instauration d'une tolérance maternelle aux alloantigènes paternels exprimés par le fœtus est indispensable à la survie de ce dernier, et donc au succès reproductif. Divers mécanismes restreignent la réponse des lymphocytes T alloréactifs maternels vis-à-vis des antigènes fœtaux: inactivation des T maternels, faible efficacité de la présentation antigénique, faible expression des molécules du CMH (complexe majeur d'histocompatibilité) par le placenta, entre autres. Compte tenu de leur importance dans la suppression des réponses immunes inappropriées ou excessives (voir les deux synthèses récemment publiées dans médecine/ sciences $[1,2]$ ) $(\rightarrow)$, l'implication

$(\rightarrow)$ Voir $\mathrm{m} / \mathrm{s} \mathrm{n}^{\circ}$ 6-7, juinjuillet 2012, page 646 et n० 8-9, août-septembre 2012, page 757 des lymphocytes $T$ régulateurs (Treg, CD $4^{+} C D 25^{+} \mathrm{FOXP3}^{+}$) dans la tolérance maternelle vis-à-vis du fœtus a naturellement été envisagée. De fait, leur nombre augmente durant la grossesse normale, et des taux bas ont été observés dans des états de prééclampsie ou des fausses-couches spontanées. Mais sans plus de précision sur le rôle de ces cellules immunosuppressives dans la réponse immune maternelle aux antigènes paternels. A. Rudensky et son équipe (University of Washington, Seattle, États-Unis) répondent à cette question en démontrant, chez la souris, que seule la sous-population des Treg induits à partir des lymphocytes $T$ $\mathrm{CD}^{+}$périphériques (pTreg) maternels, et non les Treg qui naissent dans le thymus (tTreg), contribuent à la tolérance fœtale. L'élégance et la rigueur de la stratégie expérimentale et de l'analyse phylogénique qui l'accompagne font de cet article publié dans Cell [3] un remarquable exemple de la subtilité dont font preuve les organismes pour assurer le succès de leur reproduction dans une finalité évolutive. On sait que l'induction de l'expression du gène Foxp3 est requise pour que les lymphocytes $T$ naïs périphériques se différencient en pTreg et que le TGF- $\beta$ (transforming growth factor) et l'acide rétinoïque y contribuent. 0 r, les auteurs ont précédemment identifié une séquence intronique de type enhancer (CNS1), en aval du gène Foxp3, qui contient des éléments de réponse 